\title{
Probleme und Perspektiven der Arbeitszeit- politik aus dem Bereich der IG Metall
}

\author{
Barbara Jentgens \\ Hilde Wagner
}

\section{GRUNDLEGENDE VERÄNDERUNG VORMALS PRÄGENDER ZEITARRANGEMENTS}

Seit nahezu zwei Jahrzehnten haben sich die Rahmenbedingungen für Arbeitszeitpolitik im Bereich der Metall- und Elektroindustrie wie auch in anderen Branchen tief greifend verändert. Ein langjähriges, relativ stabiles und kohärentes Regime von Arbeitsorganisation und Zeit ist offenkundig zu Ende gegangen. Die neuen Entwicklungslinien, die in diesem Heft eindrücklich beschrieben werden, ${ }^{1}$ sind mit vollständig neuen Problemlagen und Herausforderungen für die IG Metall und andere Einzelgewerkschaften verbunden.

Infolge des grundlegenden Umbruchs von Ökonomie und Arbeit, der in den 1990er Jahren manifest wurde, kehrte sich in der Metall- und Elektroindustrie der jahrzehntelange Trend zu kürzeren tariflichen und effektiven Arbeitszeiten um. Die effektiven Arbeitszeiten stiegen auch in der Metall- und Elektroindustrie deutlich an. Dabei schlug sich der Wandel nicht nur in der Entwicklung der tatsächlichen Arbeitszeiten, sondern auch in der tariflichen Arbeitzeitpolitik nieder. Die 1990er Jahre standen zwar noch im Zeichen der Umsetzung der vorher vereinbarten tariflichen Verkürzungsschritte, ohne allerdings noch eine Sogwirkung auf die effektiven Arbeitszeiten ausüben zu können. Die Linien der tariflichen und effektiven Arbeitszeiten entwickelten sich in diesem Jahrzehnt in der Metall- und Elektroindustrie stärker als in den anderen Branchen auseinander. Außerdem wurde die Verkürzung der tariflichen Wochenarbeitszeit häufig mit einer höheren Flexibilität der betrieblichen Arbeitszeitgestaltung „bezahlt“. In den vergangenen Jahren kam es darüber hinaus zu konditionierten Erhöhungsmöglichkeiten der Arbeitszeit in den Flächentarifverträgen.

Diese Entwicklungen markierten das Ende eines historischen Zeitarrangements, das als „fordistisches Zeitregime“ in die prägende Organisation und Steuerung von Arbeit und Unternehmen in den Nachkriegsjahrzehnten eingebettet war. Im Zuge der verschärften globalisierten Konkurrenz geriet auch die Arbeitszeitlandschaft in Bewegung. Die prioritäre Ausrichtung der Unternehmen an den Renditeerwartungen der Finanzmärkte bzw. an der Wertsteigerung für die Anteilseigner führt zu Renditevorgaben, die mit den mittelund langfristigen Potenzialen der Unternehmen nicht mehr viel zu tun haben. Eine „Ökonomie der kurzen Fristen“ hält Einzug, die nach innen in erster Linie mit Maßnahmen zur Arbeitskostensenkung verbunden wird. Aus Unternehmenssicht bietet sich gerade bei der Arbeitszeit ein günstiger Hebel, um den verschärften Druck auf die Arbeitskosten an die Beschäftigten weiterzugeben.

In den Betrieben geht der Umbruch mit einer historisch neuen Form der Steuerung von Arbeit einher. ${ }^{2}$ Die Beschäftigten und ihre Betriebsräte werden mit den Wettbewerbs- und Verwertungsbedingungen der Unternehmen konfrontiert. Sie geraten in eine Situation, in der sie für das Überleben im Wettbewerb und damit für die $\mathrm{Zu}$ kunft ihres Standorts und Arbeitsplatzes selbst Sorge tragen müssen. Unter den Vorzeichen indirekter Steuerung wird Arbeit tendenziell maßlos, Arbeitszeit und Leistung werden „entgrenzt“. Flexiblere Arbeitszeiten und neue „Arbeitszeit-Freiheiten" für Beschäftigte führen unter diesen Bedingungen zu dem Phänomen „Arbeiten ohne Ende“. Gleichzeitig nehmen Schicht-, Sonn- und Feiertagsarbeit wieder zu.

\section{ETAPPEN UND SCHWERPUNKTE DER ARBEITSZEITPOLITIK IN DER METALLINDUSTRIE}

\section{DIE 35-STUNDEN-WOCHE UNTER DRUCK}

Die tarifvertragliche Arbeitszeit in der Metall- und Elektroindustrie basiert nach wie vor auf der 35-Stunden-Woche. Diese wur- de 1984 vereinbart und bis 1994 stufenweise in den Betrieben eingeführt. Zuvor gab es in der Metall- und Elektroindustrie über mehr als drei Jahrzehnte kontinuierliche Arbeitszeitverkürzungen.

Bereits bei der Umsetzung der 35-Stunden-Woche erlaubte der Tarifvertrag auch andere Formen der betrieblichen Gestaltung und Verkürzung der Arbeitszeit als täglich kürzere Arbeitszeiten. Die individuelle regelmäßige wöchentliche Arbeitszeit konnte gleichmäßig oder ungleichmäßig auf Montag bis Freitag oder mehrere Wochen verteilt werden - insgesamt musste sie im Durchschnitt 35 Stunden ergeben. Dabei erfolgte die Gestaltung der Arbeitszeit zunehmend über Arbeitszeitkonten. Diese brachen mit dem lange Jahre geltenden Prinzip, die vereinbarte Regelarbeitszeit in "gleichförmigen Portionen" auf die Wochentage und das Jahr zu verteilen.

Um die zunehmende Entgrenzung der Arbeitzeit, vor allem in Bereichen mit hoch qualifizierten Beschäftigten einzufangen, wurde für die Metall- und Elektroindustrie bereits 1990 eine Öffnungsklausel vereinbart, die für $13 \%$ bzw. $18 \%$ der Beschäftigten eine bezahlte Verlängerung der Arbeitszeit auf bis zu 40 Stunden einzelvertraglich ermöglicht. Im Jahr 2004 wurde

\begin{tabular}{ll}
\hline 1 Vgl. als Überblick den Beitrag von Jürgens in die- \\
sem Heft. \\
\hline 2 Wagner, H. (Hrsg.) (2005): Rentier ich mich noch? \\
Neue Steuerungskonzepte im Betrieb, Hamburg \\
und insbesondere: Peters, K.; Sauer, D.: Indirekte \\
Steuerung - eine neue Herrschaftsform, ebd. \\
S. 23-58.
\end{tabular}

Barbara Jentgens, Ressort Grundsatzfragen im Funktionsbereich Tarifpolitik beim Vorstand der IG Metall. e-mail: barbara.jentgens@igmetall.de Hilde Wagner, Dr., Ressort Grundsatzfragen im Funktionsbereich Tarifpolitik beim Vorstand der IG Metall. e-mail: hilde.wagner@igmetall.de 
diese Vereinbarung im „Pforzheimer Abkommen " zunächst in Baden-Württemberg und dann auch in den anderen Bezirken der IG Metall erweitert: Durch freiwillige Betriebsvereinbarungen ist es seither möglich, in Betrieben mit einem Anteil von mehr als 45 bzw. $50 \%$ der Beschäftigten in den obersten Gehaltsgruppen die durchschnittliche betriebliche Arbeitszeit für maximal $50 \%$ der Beschäftigten auf bis zu 40 Stunden zu erhöhen.

Der Kern des „Pforzheimer Abkommens" bezieht sich jedoch nicht vorrangig auf das Thema Arbeitszeit. Schon vor dem Pforzheimer Abkommen stieg die Anzahl betrieblicher Regelungen zu Arbeitszeit und Einkommen deutlich an. Das Abkommen zielte darauf, die Regelungshoheit als Tarifvertragspartei zu erhalten und Tarifflucht zu begegnen. Es regelt die Voraussetzungen und Bedingungen, um vom Flächentarifvertrag abweichende Vereinbarungen treffen zu können, die „unter Abwägung der sozialen und wirtschaftlichen Folgen (...) eine nachhaltige Verbesserung der Beschäftigungsentwicklung" sichern sollen. Wenn die definierten Bedingungen vorliegen, können die Tarifvertragsparteien - nach gemeinsamer Prüfung mit den Betriebsparteien - ergänzende oder abweichende Tarifregelungen vereinbaren ( $\mathrm{z}$. B. Kürzung von Sonderzahlungen, Stundung von Ansprüchen, Erhöhung oder Absenkung der Arbeitszeit mit oder ohne vollen Lohnausgleich - soweit nicht durch Beschäftigungssicherungstarifverträge geregelt).

Um zu verhindern, dass die Betriebe dabei im Wettbewerbskarussel gegeneinander ausgespielt werden und die Standards weiter sinken, wurden innerhalb der IG Metall inhaltliche Grundsätze und Vorgehensweisen für abweichende Vereinbarungen verabschiedet. Arbeitszeitverlängerungen sollten die Ausnahme bleiben und in jedem Fall gesondert begründet werden. Aber allen Anstrengungen in den Bezirken und den Verwaltungsstellen der IG Metall und in den Betrieben zum Trotz standen im Rahmen der über 900 abweichenden Regelungen seit 2004 (in Firmen-, Sozial-, Sanierungs-, und Tarifverträgen nach "Pforzheim") veränderte Arbeitszeiten in $62 \%$ der Fälle an erster Stelle. Ein Großteil dieser Verträge bezieht sich auf Betriebe ,in wirtschaftlicher Not" (ca. 70 \% der Fälle sind Sanierungstarifverträge). Zugenommen haben aber auch die Begehrlichkeiten von Unternehmen, die Abweichungen der
Entgelte und Arbeitszeiten vom Flächentarifvertrag unabhängig von einer wirtschaftlichen Notlage „zur Verbesserung der zukünftigen Wettbewerbsfähigkeit" einfordern. Hinzu kommt, dass seitens der Beschäftigten längere Arbeitszeiten oftmals hingenommen werden, weil sie Angst davor haben, ihren Job zu verlieren oder weil sie diese einer direkten Einkommenskürzung vorziehen, oder weil die eigenen Arbeitszeiten unter den Bedingungen indirekter Steuerung bereits ausgeufert sind.

Insbesondere aufgrund der eingangs beschriebenen Entwicklungen und der verlängerten effektiven Arbeitszeiten ist die 35-Stunden-Woche als Regelarbeitszeit massiv unter Druck geraten. Auch die Versuche, sie in den anderen Organisationsbereichen der IG Metall flächendeckend einzuführen und umzusetzen, waren im vergangenen Jahrzehnt nur wenig erfolgreich. Eine schmerzvolle Niederlage musste die IG Metall 2003 vor allem beim Kampf um die Angleichung der tariflichen Arbeitszeiten in Ostdeutschland an die 35-StundenWoche im Westen hinnehmen.

\section{FLEXIBILISIERUNG DER ARBEITSZEIT}

Arbeitszeitverlängerung und Arbeitszeitflexibilisierung sind längst zum strategisch integrierten Programm der Arbeitgeber und ihrer Verbände geworden. Schon im Laufe der 1990er Jahre wurden Dauer, Lage und Verteilung der betrieblichen Arbeitszeit immer mehr an die produktionsspezifischen, konjunkturellen und marktbezogenen Erfordernisse angepasst. Seit einigen Jahren versuchen die Arbeitgeber dabei verstärkt, die weitere Flexibilisierung als Instrument einer schleichenden Arbeitszeitverlängerung meist ohne Entgeltausgleich zu nutzen. Um dieser Entwicklung entgegenzuwirken, hat die IG Metall die tarifliche Gestaltung von Arbeitszeitkonten auf die Tagesordnung gesetzt.

Die Flexibilisierung der Arbeitszeit ist in der Metall- und Elektroindustrie bereits weit fortgeschritten. Arbeitszeitkonten sind hier generell weiter verbreitet als in der Gesamtwirtschaft. Bereits im Jahr 2002 arbeiteten $55 \%$ der Beschäftigten in der Metallund Elektroindustrie mit einem Arbeitszeitkonto, ${ }^{3}$ während dies in der Gesamtwirtschaft laut einer ISO Untersuchung im Jahr 2003 für $42 \%$ der westdeutschen und $38 \%$ der ostdeutschen Beschäftigten der Fall war. Arbeitszeitkonten werden vornehmlich eingesetzt, um den Ausgleich für
Mehrarbeitsstunden zu verwalten und um eine variable Arbeitszeitverteilung zu regulieren.

Befragungen in einzelnen Bereichen und Betrieben der IG Metall ergaben, dass Arbeitszeitkonten beliebte Instrumente der Arbeitszeitgestaltung insbesondere von größeren und großen Betrieben sind. Dies bestätigt eine Arbeitszeiterhebung in einem Bezirk der IG Metall aus dem Jahr 2004/ 2005 mit dem eindeutigen Befund: Je gröBer die Betriebe, umso eher verfügen diese über Zeitkonten. Am meisten verbreitet sind demnach Gleitzeitregelungen. Über $60 \%$ der befragten Betriebe mit über 100 Beschäftigten verfügen über diese Form eines Arbeitszeitkontos. Der Anteil der Betriebe mit Langzeitkonten ist geringer, ca. $30 \%$ der Betriebe mit mehr als 500 Beschäftigten haben in diesem Bezirk Langzeitkonten. Meist werden die Langzeitkonten aus Mehrarbeitszeiten gespeist. Die Verwendung der angesparten Zeiten erfolgt in knapp $60 \%$ der Fälle für Freistellung und in einem Viertel der Fälle für die Altersteilzeit, während rund ein Drittel der Zeiten zur Auszahlung kommt. Diese Umfrage ergab auch, dass es in einer Reihe der Betriebe keine Insolvenzsicherung für betriebliche Langzeitkonten gibt.

Ein zentrales Problem besteht darin, dass angesparte Zeitguthaben auf Arbeitszeitkonten häufig verfallen. Dies ist der WSI Betriebsrätebefragung von 2004/2005 zufolge in gut einem Drittel der Betriebe mit Zeitkonten der Fall. Ein Grund hierfür kann darin liegen, dass manche Arbeitszeitkonten keine Ausgleichsregelungen beinhalten. ${ }^{4}$ Langzeit- und Lebensarbeitszeitkonten sind eher ein junges arbeitszeitpolitisches Instrument. Nach einer Befragung der Sozialforschungsstelle Dortmund verfügen gesamtwirtschaftlich $6,9 \%$ der Betriebe über ein Langzeitkonto und hochgerechnet rund $18 \%$ der Beschäftigten arbeiten in Betrieben, die ein Langzeitkonto führen. ${ }^{5}$

\footnotetext{
3 Vgl. zu den genauen statistischen Angaben die Ergebnisse einer von Gesamtmetall in Auftrag gegebenen Beschäftigtenbefragen des Instituts Demoskopie Allensbach aus dem Jahr 2002 sowie die Studie des ISO Köln: Bauer, F. et al. (2004): Arbeitszeit 2003, Köln, S. 20.

4 Vgl. u. a. Seifert, H. (2005): Vom Gleitzeit- zum Langzeitkonto, in: WSI Mitteilungen 6, S. 312.

5 Hildebrandt, E./Wotschack, P. (2006): Langzeitkonten und Lebenslaufpolitik, in: WSI-Mitteilungen 11, S. 592
} 


\begin{tabular}{|c|c|c|c|}
\hline \multicolumn{4}{|c|}{$\begin{array}{l}\text { Übersicht 1: Tarifvertragliche Regelungen über Langzeitkonten in den Tarifbezirken Baden-Württemberg, } \\
\text { Nordrhein-Westfalen und Stahl (Auszüge) }\end{array}$} \\
\hline \multirow[t]{2}{*}{ Inhalt } & Tarifbezirk & Tarifbezirk & Branche \\
\hline & Baden-Württemberg (2005) & Nordrhein-Westfalen (2005) & Stahl (2001) \\
\hline Einführung & $\begin{array}{l}\text { - durch freiwillige Betriebsvereinbarung } \\
\text { (BV) }\end{array}$ & - durch freiwillige Betriebsvereinbarung & $\begin{array}{l}\text { - durch freiwillige Betriebsvereinbarung, } \\
\text { für vorübergehende oder vorzeitige Frei- } \\
\text { stellung aus dem aktiven Beschäftigungs- } \\
\text { verhältnis }\end{array}$ \\
\hline Führung der Konten & $\begin{array}{l}\text { - in "Zeit"; eine Betriebsvereinbarung } \\
\text { kann abweichend "Geld" vorsehen }\end{array}$ & $\begin{array}{l}\text { - in "Zeit" oder "Geld“; ; eine Festlegung } \\
\text { erfolgt durch die Betriebsparteien }\end{array}$ & - Langfristkonten in „Geld“ \\
\hline $\begin{array}{l}\text { Zuführung zu den } \\
\text { Konten }\end{array}$ & $\begin{array}{l}\text { - Regelung in Betriebsvereinbarung } \\
\text { - Arbeitsstunden für die ein Vergütungs- } \\
\text { anspruch besteht } \\
\text { - Regelüberlauf aus anderen Arbeitszeit- } \\
\text { konten ist ausgeschlossen }\end{array}$ & $\begin{array}{l}\text { - Regelung in Betriebsvereinbarung } \\
\text { - Stunden für die Anspruch auf bezahlte } \\
\text { Freistellung besteht, können statt Inan- } \\
\text { spruchnahme individuell zugeführt werden } \\
\text { - Bestandteile des Arbeitsentgelts können } \\
\text { zugeführt werden (z. B. Sonderzahlungen) } \\
\text { Ausnahmen: Mehrarbeitsvergütungen bei } \\
\text { Gesamtzuführung > } 152 \text { Std./Jahr und } \\
\text { steuer-/sozialversicherungsfreie Entgelte } \\
\text { - Urlaubs- und Sonderzahlungsansprüche } \\
\text { bleiben unverändert oder werden gleich- } \\
\text { wertig abgegolten } \\
\text { - Festlegung durch BV, ob Ansprüche auf } \\
\text { Urlaub oder betriebliche Sonderzahlungen } \\
\text { in der Entnahmephase oder ob Ansprüche } \\
\text { mit Hilfe eines Bewertungsfaktors }(1,25) \\
\text { bereits beim Einbuchen (Zuführung) } \\
\text { berücksichtigt werden } \\
\text { - Regelüberlauf aus anderen Arbeitszeit- } \\
\text { konten ist ausgeschlossen }\end{array}$ & $\begin{array}{l}\text { - zum Aufbau von Wertguthaben können, } \\
\text { ggf. nach Umrechnung in den aktuellen } \\
\text { Geldwert, die im Folgenden beschriebenen } \\
\text { Leistungen verwendet werden: } \\
\text { Sonderzahlungen (tarifliche und betrieb- } \\
\text { lich), Teile des Monatsentgelts, Variable } \\
\text { Lohn- und Gehaltsbestandteile, Mehr- } \\
\text { arbeitsgrundvergütungen, Zuschläge für } \\
\text { Mehrarbeit, Vermögenswirksame Leistun- } \\
\text { gen, Nachtfreischichten, nach betrieblicher } \\
\text { Regelung Guthaben für Arbeitszeit aus } \\
\text { nicht gemäß § } 1 \text { Ziffer } 7 \text { des Tarifvertrages } \\
\text { zur Beschäftigungssicherung und zur } \\
\text { Einführung von Arbeitszeitkonten ausge- } \\
\text { glichenen Arbeitszeitkonten, soweit diese } \\
\text { Guthaben nicht auf Mehrarbeit beruhen } \\
\text { (multipliziert mit 1,23) }\end{array}$ \\
\hline Grenzen der Zuführung & $\begin{array}{l}\text { - } 152 \text { Stunden pro Jahr, außer aus IRWAZ } \\
\text { sofern eine Betriebsvereinbarung dies } \\
\text { regelt }\end{array}$ & - 152 Stunden pro Jahr & $\begin{array}{l}\text { Für Zeitanteile darf pro Jahr max. der } \\
\text { Geldwert von } 169 \text { Stunden aufgebucht } \\
\text { werden. Insgesamt dürfen Beschäftigte } \\
\text { pro Jahr max. } 15 \% \text {, ab Vollendung des } \\
\text { 45. Lebensjahres } 20 \% \text { ihres Brutto- } \\
\text { entgelts aufbuchen. }\end{array}$ \\
\hline Entnahme & $\begin{array}{l}\text { - Entscheidung des Beschäftigten: } \\
\text { - Vor Rentenzugang entsprechende } \\
\text { Entnahme bis Renteneintritt, } \\
\text { - in der Arbeitsphase für Altersteilzeit, } \\
\text { - Persönliche Weiterbildung im Sinne §5 } \\
\text { Tarifvertrag Qualifizierung } \\
\text { Diese Verwendungszwecke können im } \\
\text { Rahmen einer BV nicht ausgeschlossen } \\
\text { werden. } \\
\text { Darüber hinaus bei Regelung durch BV } \\
\text { zusätzlich: } \\
\text { - Abkürzung der gesetzlichen Elternzeit, } \\
\text { - sonstige, wenn Arbeitgeber und } \\
\text { Beschäftigte sich darauf einigen }\end{array}$ & $\begin{array}{l}\text { - Entscheidung des Beschäftigten im } \\
\text { Rahmen der vereinbarten Ziele nach } \\
\text { Maßgabe der Betriebsvereinbarung und } \\
\text { der folgenden Bestimmungen: } \\
\text { - Freistellung vor Rentenbeginn, } \\
\text { - Freistellung in der Arbeitsphase der } \\
\quad \text { Altersteilzeit }\end{array}$ & $\begin{array}{l}\text { Die Wertguthaben werden zur Finanzie- } \\
\text { rung steuer- und sozialversicherungs- } \\
\text { pflichtiger Freistellungsphasen verwendet. } \\
\text { Deren Lage wird vom Beschäftigten unter } \\
\text { Berücksichtigung der betrieblichen Belange } \\
\text { und des Zwecks der Langfristkonten } \\
\text { bestimmt. Die Dauer des Anspruchs auf } \\
\text { Freistellung wird auf der Basis des zuletzt } \\
\text { erzielten Monatsentgelts und der entspre- } \\
\text { chenden Sozialversicherungsbeiträge } \\
\text { errechnet. } \\
\text { Durch Individualvereinbarung kann der } \\
\text { Freistellungsanspruch verlängert werden. } \\
\text { Die Freistellungsphase kann auch in der } \\
\text { Arbeitsphase einer vereinbarten Alters- } \\
\text { teilzeit liegen. }\end{array}$ \\
\hline $\begin{array}{l}\text { Ankündigungsfristen } \\
\text { für Entnahme }\end{array}$ & $\begin{array}{l}\text { - Regelung in BV, nicht unter einem } \\
\text { Monat } \\
\text { - besondere Fristen bei Entnahme für } \\
\text { Qualifizierung }\end{array}$ & $\begin{array}{l}\text { - Regelung in BV, aber nicht unter einem } \\
\text { Monat }\end{array}$ & \\
\hline Insolvenzsicherung & $\begin{array}{l}\text { - ab der 1. Stunde } \\
\text { - BV wird erst wirksam mit Nachweis der } \\
\text { Insolvenzsicherung; Sicherungsformen } \\
\text { gem. § 8a ATZG. Umfang It. stichtags- } \\
\text { bezogener Feststellung halbjährlich }\end{array}$ & $\begin{array}{l}\text { - ab der 1. Stunde, ab dem 1. Euro } \\
\text { - BV wird erst wirksam mit Nachweis der } \\
\text { Insolvenzsicherung. Sicherungsformen } \\
\text { gem. § 8a ATZG. Umfang It. stichtags- } \\
\text { bezogener Feststellung halbjährlich }\end{array}$ & $\begin{array}{l}\text { Der Arbeitgeber gewährleistet, dass im } \\
\text { Falle einer Insolvenz jedes Wertguthaben } \\
\text { gesichert ist. Er weist gegenüber dem } \\
\text { Betriebsrat jährlich die ausreichende } \\
\text { Sicherung nach. }\end{array}$ \\
\hline $\begin{array}{l}\text { Auszahlungsregeln } \\
\text { bei Störfällen }\end{array}$ & $\begin{array}{l}\text { - bei Tod } \\
\text { - bei Beendigung des Arbeitsverhältnisses, } \\
\text { wenn kein Abbau des Kontos möglich ist } \\
\text { - auch in bAV, wenn Rentenabschläge } \\
\text { ausgeglichen werden und BV dies vorsieht }\end{array}$ & $\begin{array}{l}\text { - bei Tod } \\
\text { - bei Beendigung des Arbeitsverhältnisses, } \\
\text { wenn kein Abbau des Kontos möglich ist }\end{array}$ & $\begin{array}{l}\text { Kann das Wertguthaben nicht gemäß } \\
\text { verwendet werden, wird es einvernehmlich } \\
\text { in eine Form bAV umgewandelt oder zur } \\
\text { Aufstockung von ATZ-Entgelt oder zum } \\
\text { Ausgleich evtl. Rentenabschläge verwen- } \\
\text { det. Ist dies nicht möglich, erfolgt eine } \\
\text { Auszahlung. } \\
\text { Bei Tod geht das Guthaben an die Erben. }\end{array}$ \\
\hline Quelle: Zusammenstellung c & nnen. & & WSI $\begin{array}{c}\text { Hans Böckler } \\
\text { Stiftung }\end{array}$ \\
\hline
\end{tabular}

In den vergangenen Jahren hat sich die IG Metall um eine tarifpolitische Gestaltung von Arbeitszeit in Form von Zeitkonten bemüht und in zwei Tarifgebieten der Metall- und Elektroindustrie (2005) und in der Stahlbranche (2001) entsprechende
Tarifverträgen abgeschlossen. Demnach können in Baden-Württemberg über freiwillige Betriebsvereinbarungen „flexible Arbeitszeitkonten“ und „Langzeitkonten“ eingeführt werden. Für beide Zeitkonten wurden dabei die Ausgleichszeiträume auf- gehoben. Beim „flexiblen Arbeitszeitkonto" sind die Regeln für die Zuführung von Zeitguthaben zwischen den Betriebsparteien zu vereinbaren. Das Konto wird als individuelles Zeitkonto geführt. Für Guthaben- bzw. Minusstunden sind Ober- und 
Untergrenze festzulegen. Wenn das Zeitguthaben 300 Stunden übersteigt, hat eine Insolvenzsicherung zu erfolgen. Die Tarifverträge über Langzeitkonten schreiben in Baden-Württemberg und in NordrheinWestfalen Betriebsvereinbarungen vor, um dieses Instrument betrieblich einzuführen. In beiden Tarifgebieten ist vorgesehen, dass das angesparte Zeitguthaben vorrangig dafür verwendet wird, vorzeitig aus dem Erwerbsleben auszusteigen oder die Arbeitsphase im Altersteilzeit-Blockmodell zu verkürzen. Auch der Tarifvertrag für die Stahlbranche sieht vor, dass Wertguthaben gebildet und in erster Linie für die vorübergehende oder vorzeitige Freistellung aus dem aktiven Beschäftigungsverhältnis entnommen werden. Die Tarifverträge erlauben aber auch weitere Anlässe und Möglichkeiten für die Entnahme von Zeiten. So besteht in Baden-Württemberg die Möglichkeit, das Zeitguthaben für die persönliche Weiterbildung zu verwenden; dies kann in Nordrhein-Westfalen optional über Betriebsvereinbarungen geregelt werden. Außerdem sind Betriebsvereinbarungen zur Freistellung im Zusammenhang mit Elternzeiten und für sonstige Verwendungszwecke möglich. Alle Tarifverträge beinhalten Obergrenzen für die zeitliche Zuführung (vgl. zu den Inhalten der Tarifverträge Übersicht 1).

\section{GESTALTUNGSAUFGABEN FÜR ARBEITS- UND LANGZEITKONTEN}

Die Tarifverträge über Arbeitszeitkonten bieten Ansatzpunkte dafür, dass und wie zusätzlich geleistete Arbeitszeiten für „Freistellungen" aus dem Erwerbsleben genutzt werden können. Diese Ansatzpunkte gilt es im Sinne einer höheren Zeitsouveränität für die Beschäftigten auszubauen. Wenn allerdings die Personalkapazitäten in den Unternehmen immer geringer bemessen werden und gleichzeitig das Arbeitsvolumen und die Arbeitsverantwortung steigt, werden auch weiterhin maßlose Arbeitszeiten mit Folgen für Gesundheit und soziales Leben nicht ausbleiben.

Arbeitszeitkonten und insbesondere Lang- und Lebensarbeitszeitkonten gehen häufig damit einher, dass Arbeitszeiten angesammelt und nicht belastungsnah ausgeglichen, sondern "geparkt" und weitertransportiert werden. Daher ist es wichtig, Rahmenbedingungen für ihre Gestaltung zu definieren. Dabei kommt es darauf an, flexible Arbeitszeiten und Konten mit Ge- staltungsspielräumen für die Beschäftigten zu verbinden. Diese können durch individuelle Verfügungsrechte in tariflichen oder betrieblichen Regelungen abgesichert werden. Folgende Punkte sollten dabei berücksichtigt werden:

- Arbeitszeiten müssen für die Beschäftigten planbar sein. Erst die Verlässlichkeit von Arbeitzeiten ermöglicht es, Arbeit und privates Leben zu synchronisieren. Hierbei ist es wichtig, Fristen zu vereinbaren, in denen das „Hoch- und Runterfahren“ von Arbeitszeiten anzukündigen ist.

- Flexible Arbeitszeiten und Arbeitszeitkonten müssen den ergonomischen Grundsätzen der Arbeitzeitgestaltung entsprechen. ${ }^{6}$ Arbeitszeiten müssen gesundheitsverträglich bleiben. Es ist deshalb nicht akzeptabel, dass überlange Arbeitszeiten einfach finanziell kompensiert werden.

- Basis für Arbeitszeitkonten müssen ein definierter Arbeitszeitrahmen sowie die 35-Stunden-Woche bilden. Dabei müssen Obergrenzen für die Zuführung festgelegt werden.

- Bei den Entnahmemöglichkeiten sind die Entscheidungsrechte der Beschäftigten zu stärken. Dies ist eine wichtige Voraussetzung für mehr Zeitsouveränität.

- Ein Regelungsüberlauf von Kurzfristkonten zu Langfristkonten ist auszuschließen.

- In den Vereinbarungen sind Sanktionsmechanismen vorzusehen, für Fälle in denen gegen tarifliche oder betriebliche Regelungen verstoßen wird.

- Langzeitkonten sind ab der ersten Stunde (bzw. ab dem ersten Euro) gegen Insolvenz zu sichern.

\section{PERSPEKTIVEN UND HANDLUNGS- FELDER ZUKÜNFTIGER ARBEITSZEITPOLITIK}

Arbeitszeiten, die es Frauen und Männern erlauben, am sozialen, kulturellen und gesellschaftlichen Leben teilzuhaben und gesund und arbeitsfähig zu bleiben, müssen auf der Tagesordnung wieder einen bedeutenderen Platz bekommen. Deshalb sind auch der Erhalt der 35-Stunden-Woche bzw. ihre weitere Einführung in anderen Branchen - und die Verkürzung bereits ausufernder Arbeitszeiten weiterhin politische Ziele. Für besonders belastete Beschäftigtengruppen, wie Schichtarbeiterinnen, müssen kürzere Arbeitszeiten umgesetzt werden. Ergänzend geht es darum, die individuellen Rechte und Optionsmöglichkeiten für die Beschäftigten sowie die Mitbestimmungsmöglichkeiten der Betriebsräte bei der Arbeitszeit zu stärken. Gewerkschaftliche Arbeitszeitpolitik hat sich insgesamt einer neuen Herausforderung zu stellen: Wenn unter dem Druck der Standortkonkurrenz und unter den Bedingungen neuer Steuerung immer mehr Beschäftigte bisher wirksame tarifliche oder betriebliche Regelungen und Instrumente nicht mehr in Anspruch nehmen, ${ }^{7}$ kommt es darauf an, die einzelnen Beschäftigten dabei zu unterstützen, selbst Grenzen zu setzen. Ein Rückgriff auf vorgegebene starre Zeitregime oder die Stechuhr ist dabei wenig hilfreich. Sinnvoller ist es, zusammen mit den Beschäftigten die Bedingungen ihrer Arbeit zu reflektieren und sie in die betriebliche und gewerkschaftliche Willensbildung und die Auseinandersetzung um eine sinnvolle Regulierung stärker einzubeziehen. Arbeitszeitinitiativen der IG Metall, wie „Arbeiten ohne Ende?“ und „Meine Zeit ist mein Leben" waren und sind Schritte in diese Richtung.

In diesem Sinne sind auch Regelungen anzustreben, die Wahlmöglichkeiten für die Beschäftigten und „Haltegriffe“ beinhalten, ${ }^{8}$ die bei Bedarf genutzt werden können. Es ist wichtig, Vereinbarungen zu treffen, die den einzelnen Beschäftigten helfen, über ihre eigenen Arbeitszeiten möglichst selbstbestimmt zu verfügen. Dies ist unerlässlich, damit Gesundheitsrisiken eingedämmt und die Wünsche der Beschäftigten nach mehr Zeitsouveränität nicht dem unternehmerischen Flexibilitätsdiktat geopfert werden.

Dabei ergeben sich folgende Handlungsfelder für die gewerkschaftliche Arbeitszeitpolitik:

Alternsgerechte und gesundheitsverträgliche Arbeitszeiten: Vor dem Hintergrund der Intensivierung der Arbeit und des demografischen Wandels sowie der kürzlich beschlossenen Anhebung des Renteneintrittsalters auf 67 Jahre verfolgt die IG Metall das Ziel, Konzepte alternsgerechten

\footnotetext{
6 Vgl. Fergen, A. (2007): Schlechte Zeiten - Gute Zeiten. Mit gewerkschaftlicher Arbeitszeitpolitik zu "guter Arbeit", in: Peter, G. (Hrsg): Grenzkonflikte der Arbeit, Hamburg (im Erscheinen).

7 Vgl. den Beitrag von Kratzer/Sauer in diesem Heft.

8 Vgl. den Beitrag von Haipeter/Lehndorff in diesem Heft.
} 
Arbeitens umzusetzen. Dieser Ansatz nimmt die gesamte Erwerbsarbeitszeit in den Blick. Dabei stehen tarifvertragliche und betriebliche Maßnahmen auf der Tagesordnung, die es den Beschäftigten gestatten, ihr Erwerbsleben gesund und qualifiziert bis zum Renteneintrittsalter bewältigen zu können. ${ }^{9}$ In der Tarifrunde Stahl 2006 waren für einen „Tarifvertrag alternsgerechtes Arbeiten“ "von Seiten der IG Metall - zusätzlich zu den erreichten Regelungen, die für alle Betriebe zum Beispiel Altersstrukturanalysen vorsehen - auch geringere Arbeitszeiten für besonders belastete Beschäftigtengruppen geplant. Diese wurden von den Arbeitgebern abgelehnt. In Zukunft werden jedoch spezifische Formen der Arbeitszeitverkürzung für gesundheitlich besonders belastete Beschäftigte, z. B. in Nacht- und Wechselschichtarbeit eine wichtigere Rolle spielen. ${ }^{10}$ Gerade in Arbeitsbereichen mit anstrengenden physischen und/oder hohen psychischen Anforderungen sollten Arbeitszeiten mit einem belastungsnahen Ausgleich ermöglicht werden. Weil nicht zuletzt wegen der gegenwärtigen Arbeits- und Leistungsbedingungen lediglich ca. 3,2 \% der Beschäftigten in der Metall- und Elektroindustrie 60 Jahre und älter sind, muss auch über das Jahr 2009 hinaus die Möglichkeit der Altersteilzeit zu materiell akzeptablen Bedingungen erhalten bleiben.

Erwerbsbiografieorientierte Arbeitszeitgestaltung: Die IG Metall strebt Modelle der Arbeitszeitgestaltung an, die sich auf die unterschiedlichen Lebensphasen und Bedürfnisse der Beschäftigten im Laufe ihrer Erwerbsbiografie beziehen. Es geht dabei um Optionsmodelle, die eine Unterbrechung der Erwerbsarbeit vorsehen, z. B. für Elternzeiten, für die Pflege von Familien- mitgliedern oder für Bildung. Erwerbsbiografieorientierte Arbeitszeiten bedeuten auch, dass während des Erwerbsverlaufs andere Regelungen als das Vollarbeitszeitverhältnis möglich sein sollen.

Balance zwischen Arbeit und Privatleben: Weil es den Beschäftigten zunehmend schwerer fällt, ihr Privatleben und ihren Beruf zu vereinbaren, will die IG Metall Arbeitzeitmodelle fördern, die es den Beschäftigten ermöglichen, ihre Arbeitszeiten so autonom zu gestalten, dass sie ihren individuellen Ansprüchen entsprechen. In diesem Zusammenhang könnten die oben erwähnten Optionsmodelle auch um weitere Konditionen ergänzt werden, wie zum Beispiel die Möglichkeiten für Sabbaticals. Um die tägliche und wöchentliche Balance nicht noch weiter aus dem Gleichgewicht geraten zu lassen, sind auch Maßnahmen erforderlich, die eine gerechtere Verteilung der Arbeitszeiten zwischen Männern und Frauen fördern.

\section{FAZIT}

Die dringlichste Aufgabe gewerkschaftlicher Arbeitszeitpolitik besteht aktuell darin, der weiteren Ausuferung und Polarisierung sowie dem „Verfall“ von Arbeitzeiten entgegenzuwirken und die Zeitsouveränität der Beschäftigten zu erhöhen. „Der Arbeitszeit ein gesundes Maß geben“- dies ist angesichts der gegenwärtigen Verschärfung der Arbeits- und Leistungsbedingungen zur Tagesaufgabe geworden. Allerdings kann diese Aufgabe nicht allein tarifpolitisch, sondern nur im Zusammenwirken mit Betriebspolitik und Gesundheitsschutz angegangen werden. Und sie wird nicht zu bewältigen sein, wenn nicht auch die Beschäftigten selbst Grenzen setzen.
Um alternsgerechte und biografieorientierte Arbeitszeiten realisieren zu können, ist in den Betrieben insgesamt eine andere Arbeits-, Leistungs- und Personalpolitik notwendig. Wirksame Schritte in diese Richtung wird es allerdings nur geben, wenn sich die Beschäftigten und Mitglieder in den Betrieben, unterstützt von der IG Metall, mit hohem Engagement für entsprechende Weichenstellungen einsetzen.

Angesichts der fortschreitenden Ökonomisierung werden alle rein arbeitszeitpolitischen Interventionen nur eine begrenzte Reichweite entfalten, wenn sie nicht in ein umfassenderes Konzept von „Guter Arbeit" eingebettet werden. Sie bedürfen außerdem einer flankierenden betrieblichen und gesellschaftlichen Debatte über Arbeits- und Lebenszeiten. „Zeit" beinhaltet immer ein emanzipatorisches Element. Denn mit ihr sind Vorstellungen über ein alternatives Arbeiten und Leben verbunden. Gerade in Zeiten, in denen Freiheitsgrade wachsen und gleichzeitig eingeschränkt werden, kommt es darauf an, die autonomen Zeitinteressen der Beschäftigten gegen die Maßlosigkeit der Konkurrenz zu unterstützen.

\footnotetext{
9 Um hierbei den betrieblichen Akteuren Hilfestellung zu geben, wurden in dem IG Metall Projekt "Gute Arbeit" Instrumente - wie der "ArbeitszeitTÜV ${ }^{\prime \prime}$ - entwickelt, die z. B. eine Gefährdungsbeurteilung von Arbeitszeiten möglich machen.

10 Zusätzlich zum "Arbeitszeit-TÜV“ wurde im Projekt "Gute Arbeit" in Zusammenarbeit mit dem TÜV Nord eine Software zur Schichtplangestaltung (OPTISCHICHT) und eine Broschüre erarbeitet, die dabei helfen sollen, arbeitswissenschaftliche Kriterien zu berücksichtigen.
} 